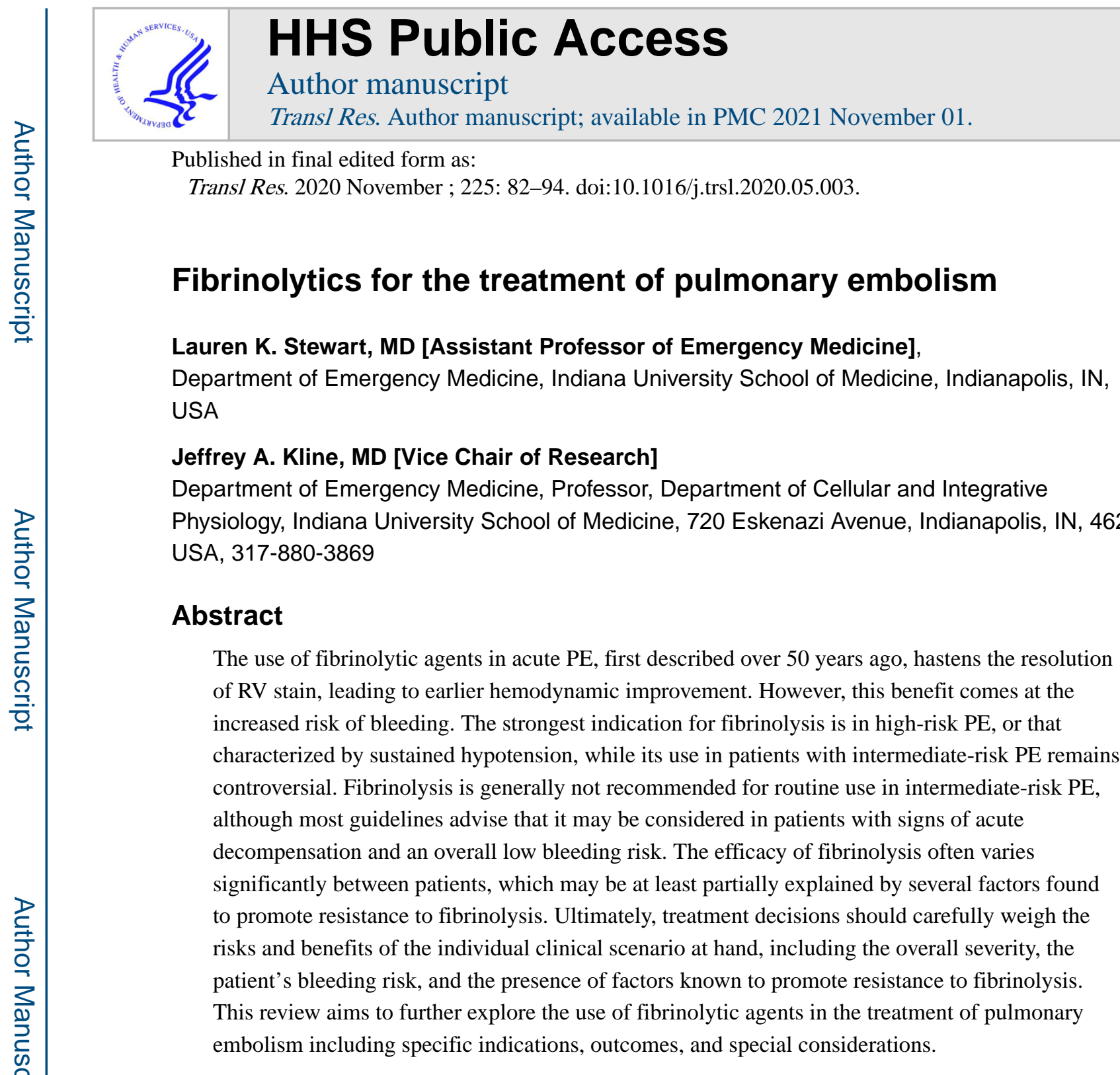

\title{
Introduction:
}

The use of fibrinolytic agents in the treatment of pulmonary embolism (PE) was first described over 50 years ago. $[1,2]$ Depending on its severity, acute PE can lead to increased pulmonary vascular resistance and right ventricular (RV) afterload, resulting in RV hypokinesis and dilation and, ultimately, RV failure. The general mechanism of fibrinolytic agents involves the activation of native plasminogen to plasmin, which hydrolyzes fibrin and leads to accelerated clot lysis.[3] The goal of this process is to reduce thrombus burden, resulting in a more rapid relief of RV afterload and earlier hemodynamic improvement. However, thrombolytic agents are not without risks. The most feared complication of systemic fibrinolysis is major bleeding, which can be life-threatening. Therefore, the use of

Corresponding author: jefkline@iu.edu.

Publisher's Disclaimer: This is a PDF file of an unedited manuscript that has been accepted for publication. As a service to our customers we are providing this early version of the manuscript. The manuscript will undergo copyediting, typesetting, and review of the resulting proof before it is published in its final form. Please note that during the production process errors may be discovered which could affect the content, and all legal disclaimers that apply to the journal pertain. 
these agents in acute PE requires a careful risk vs. benefit analysis. Clinical guidelines generally all recommend the use of systemic fibrinolysis in patients with "high-risk" PE, characterized by sustained hypotension. Its use in patients with "intermediate-risk" PE remains controversial.[4-6] The efficacy of fibrinolysis often varies significantly between patients, which may be at least partially explained by several factors found to promote resistance to fibrinolysis. This review aims to further explore the use of fibrinolytic agents in the treatment of pulmonary embolism including specific indications, outcomes, and special considerations.

\section{Fibrinolytic agents:}

All commercially available fibrinolytic agents work through the activation of plasminogen, a zymogen that requires modification in order to have proteolytic activity on the fibrin backbone. These agents, with the exception of streptokinase, covalently cleave plasminogen to form plasmin, which then hydrolyzes insoluble fibrin into soluble polypeptide derivatives, reducing the thrombus size. Streptokinase instead functions by binding to plasminogen, resulting in a conformational change that sterically opens its serine protease active sites and greatly increases its catalytic activity.[3, 7-11] Currently, there are three fibrinolytic agents approved by the FDA with an indication for use in acute PE: alteplase (rt-PA), streptokinase and urokinase. Although not cleared by the FDA, the use of tenecteplase and reteplase, two genetically modified versions of rt-PA, has also been described in the treatment of PE. Specific properties of these fibrinolytic agents, including dosing guidelines, are displayed in Table 1.[12-14]

\section{First-generation agents}

Streptokinase and urokinase are both considered to be first-generation fibrinolytic agents. Despite being cleared by the FDA for use in acute PE, these agents have limited availability in the United States. Streptokinase is a bacterial polypeptide produced from beta-hemolytic streptococcus cultures that binds to plasminogen, creating a streptokinase-plasminogen activator complex. It is antigenic and carries with it a higher risk of allergic reactions than other agents. $[9,15,16]$ The use of urokinase in PE, which is formed in the kidneys and excreted in the urine, was reported in 1970 in the landmark UPET trial. This study demonstrated urokinase to lead to a more complete resolution of pulmonary vascular obstruction at 24 hours compared to placebo, although there appeared to be no difference in overall mortality.[17] Under normal conditions, urokinase is primarily involved in extravascular remodeling and repair after tissue injury.[18] Both of these first-generation thrombolytic agents lack fibrin specificity, as they bind circulating systemic plasminogen and fibrin-bound plasminogen with similar affinity, resulting in the depletion of fibrinogen concentrations. Overall, this lack of fibrin specificity coupled with the longer infusion durations and higher risk of adverse reactions limits the widespread use of first-generation agents.[7, 8]

\section{Second-generation agents}

Alteplase (rt-PA) is an enzyme manufactured by recombinant DNA technology to resemble tissue plasminogen activator (t-PA), a naturally occurring serine protease. Unlike the first- 
generation agents, alteplase is relatively fibrin specific. In order to complete its two-step cleavage to plasmin, alteplase must co-localize with plasminogen on fibrin, thus preferentially activating plasminogen present on the thrombus surface itself over systemic plasminogen. The benefit of this increased fibrin specificity is the reduction of undesired digestion of soluble fibrinogen and a more focused fibrinolysis within the lung vasculature. $[3,9,19]$

\section{Third-generation agents}

The off-label use of tenecteplase and reteplase, two third-generation, genetically modified versions of rt-PA, has been reported in the treatment of PE. One prominent example of this is the use of tenecteplase in the PEITHO trial, the largest randomized, placebo-controlled trial of fibrinolysis for the treatment of PE to date.[14] These third-generation agents have been specifically modified to evade binding to plasminogen activator inhibitor 1 (PAI-1), which is the natural inhibitor of fibrinolysis. This modification creates longer half-lives than prior generation agents, allowing for the use of bolus dosing.[20, 21]

\section{Adverse reactions:}

The most common adverse event of fibrinolytic agents is bleeding, which can vary widely in severity, ranging from minor bleeding (e.g. bleeding from puncture wounds) to lifethreatening bleeding (e.g. intracranial hemorrhage [ICH]). Despite the relatively short halflives of the fibrinolytic agents included in Table 1, it is important to note that the risk of clinically significant bleeding generally remains elevated for a period of 12 to 24 hours after cessation of the thrombolytic infusion. There is a higher risk of major bleeding associated with systemic fibrinolysis versus standard anticoagulation alone. Pooled data suggest a $0-33 \%$ range in frequency of major bleeding in patients treated with systemic fibrinolysis, with a 0-7.4\% rate of the dreaded ICH.[22] Data from the PEITHO trial, the largest randomized, placebo-controlled trial of fibrinolysis for intermediate-risk PE to date, demonstrated a $2.0 \%$ incidence of ICH in patients receiving tenecteplase, all of whom were 65 years of age or older. Major extracranial bleeding rate in PEITHO patients receiving tenecteplase was $11.1 \%$ in patients older than 75 compared to $4.1 \%$ in those patients aged 75 and younger.[14] Several predictors of bleeding following thrombolytic administration have been identified. These include the administration of catecholamines for systemic arterial hypotension, a prolonged prothrombin time, recent major surgery, low body weight and diabetes mellitus.[23, 24] Although not yet independently validated, the PE-CH score was developed to help predict the risk of ICH in patients with PE treated with thrombolytic therapy. This risk score includes the following four prognostic factors: pre-existing Peripheral vascular disease ( 1 point), age greater than 65 years (Elderly) (1 point), prior Cerebrovascular accident with residual deficit ( 5 points), and prior myocardial infarction (Heart attack) (1 point). Scores of 0, 1, 2 and $\geq 5$ points were associated with ICH risks of $1.2 \%, 1.9 \%, 2.4 \%$ and $17.8 \%$, respectively.[25] In addition to bleeding, fibrinolytic agents may also result in allergic reactions, with the majority being related to administration of streptokinase. These events can range from mild reactions such as rash and pyrexia (up to $10 \%$ ) to major reactions, such as anaphylaxis, which are much less common (less than $0.5 \%)$. Streptokinase may also cause hypotension. $[15,16,26]$ In rare circumstances, 
alteplase has been reported to cause angioedema and anaphylactoid reactions, which tend to be mild and self-limited.[27, 28]

\section{Contraindications:}

There are several commonly recognized contraindications to systemic fibrinolysis, which are classified as either absolute or relative. Absolute contraindications include active bleeding, prior history of intracranial hemorrhage, brain or spinal surgery within the past month, ischemic stroke within the past three months, suspected aortic dissection, structural cerebrovascular disease or CNS neoplasm, recent head trauma with fracture or brain injury, and abdominal surgery within the past seven days. Several relative contraindications also exist, which indicate to providers that particular caution should be used as these patients are at higher risk of developing a complication. These contraindications are listed in Table 2.[4, 5] The bleeding risk for each individual patient and the weight of these contraindications should ultimately be considered on a case-by-case basis dependent on the severity of the specific clinical scenario at hand. In patients diagnosed with high-risk PE and determined to have a contraindication to fibrinolytic therapy, alternative treatment options may be considered including surgical embolectomy, catheter-directed thrombectomy and mechanical circulatory support.

\section{Approach to bleeding:}

Many providers believe that an inverse correlation exists between bleeding frequency and the nadir of the circulating fibrinogen concentration after thrombolysis.[29, 30]

Administration of bolus tenecteplase for PE produces trivial decreases in fibrinogen at 24 hours compared with placebo, suggesting little value in measuring fibrinogen concentration with tenecteplase.[31] Bolus dose alteplase produces significantly less loss of fibrinogen than does a 2-hour infusion regimen over the subsequent 6-8 hours, but both recover within $20-30 \%$ of baseline values at 24 hours.[32] It is somewhat puzzling to observe in practice that low dose infusion of alteplase via catheter directed thrombolysis (CDT) over 24 hours appears to produce a 50-60\% greater fibrinogenolytic effect than larger doses of plasminogen activators administered systemically over a shorter time frame. It is perhaps for this reason that the majority of experts surveyed monitor the fibrinogen concentration during CDT and stop the infusion when fibrinogen levels drop below a prespecified threshold.[33] However, after controlling for infusion dose, the direct evidence between hypofibrinogenemia and bleeding with CDT remains uncertain.[29, 34] Taken together, the results of our review do not suggest an evidence-based case to monitor fibrinogen with thrombolytic therapy, regardless of dose or route, but personal preferences are likely to supersede the evidence.

In the case of severe bleeding with thrombolytic therapy, almost no comparative evidence exists to guide hemostatic treatment options. Unfortunately, empirical logic must guide the choice of pharmacological interventions (deliberately excluding the discussion of direct mechanical vascular control of bleeding, surgical or endovascular interventions). The first objective is to stop all infusions of fibrinolytic and anticoagulants. Second is to reverse what can be reversed, usually starting with protamine (e.g., $1 \mathrm{mg} / \mathrm{kg}$ intravenously) if the patient 
has received any form of heparin. However, "reversing" the effect of plasminogen activators remains in the realm of expert opinion. Theoretically, a $10-15 \mathrm{mg} / \mathrm{kg}$ dose of rapidly administered intravenous tranexamic acid should at least partially inactivate circulating and fibrin-bound plasmin. Other options include infusions of plasma, cryoprecipitate, and commercial concentrates of clotting proteins, such as K-Centra®. However, no controlled trial has demonstrated the effectiveness of hemostatic agents in the setting of acute bleeding after thrombolytic therapy.

\section{Indications:}

Acute pulmonary embolism can be stratified based on severity into high-risk, intermediaterisk, and low-risk groups, the criteria for which are displayed in Table 3.[4-6] This section discusses the specific indications for use of thrombolytic therapy in each of these groups.

\section{Thrombolytic therapy in high-risk PE}

High-risk PE, previously referred to as massive PE, is defined by sustained hypotension (systolic blood pressure $<90 \mathrm{mmHg}$, or a drop of at least $40 \mathrm{mmHg}$ from baseline, for at least 15 minutes or requiring inotropic support, not secondary to another cause), signs or symptoms of shock, or pulselessness. Common signs or symptoms of shock may include pale or cyanotic skin, diaphoresis, acute delirium or the use of accessory muscles. High-risk $\mathrm{PE}$ is associated with a 24-hour short-term mortality rate $>20 \%$.[35, 36] Overall data supporting the use of thrombolytic therapy in high-risk PE are limited as there are no large, randomized controlled trials directly investigating fibrinolysis in unstable patients. However, pooled data from several systematic reviews and meta-analyses suggest an increased survival benefit with thrombolytic therapy when used in patients with sustained hypotension or cardiogenic shock.[37-48] Despite the relative paucity of evidence, high-risk PE is the clearest indication for fibrinolysis with all major guidelines generally agreeing that the potential benefit likely outweighs the risk of harm in this group. Specifically, this recommendation is consistent with guidelines sponsored by the American College of Chest Physicians ( $2 \mathrm{~B}=$ weak recommendation based on moderate quality evidence), the American Heart Association ( $\mathrm{IIaB}=$ moderate recommendation based on moderate quality evidence), and the European Society of Cardiology $(1 \mathrm{~B}=$ general agreement based on moderate level of evidence).[4-6]

\section{Thrombolytic therapy in intermediate-risk PE}

Intermediate-risk, or submassive, $\mathrm{PE}$ is defined by normotension and signs of RV dysfunction or abnormal cardiac biomarkers. Imaging findings consistent with RV dysfunction include either RV dilation (apical 4-chamber RV diameter divided by LV diameter >0.9) or RV systolic dysfunction on echocardiography, or RV dilation on computed tomography. Abnormal biomarkers suggestive of RV dysfunction or myocardial necrosis include elevation of any of the following: BNP $(>90 \mathrm{pg} / \mathrm{mL})$, N-terminal pro-BNP $(>500 \mathrm{pg} /$ $\mathrm{mL}$ ), troponin $\mathrm{I}(>0.4 \mathrm{ng} / \mathrm{mL})$, troponin $\mathrm{T}(>0.1 \mathrm{ng} / \mathrm{mL})$, or high precision troponin $\mathrm{T}(>14$ $\mathrm{ng} / \mathrm{mL})$. $[6,49]$ As intermediate-risk PE can be a rather heterogenous group, some have suggested further dividing this group into intermediate-high and intermediate-low risk categories (Table 3). The role for fibrinolysis in intermediate-risk PE remains controversial 
and unclear due in part to this group's large heterogeneity. Generally, societal guidelines do not recommend fibrinolysis for routine use in intermediate-risk PE. However, most guidelines advise that fibrinolysis may be considered in patients with signs of acute decompensation and an overall low bleeding risk.[4-6] This may include patients with new hemodynamic instability such as increasing tachycardia or shock index, worsening respiratory insufficiency, severe RV dysfunction, or major myocardial necrosis. Several recent randomized controlled trials have studied the use of fibrinolysis in intermediate-risk PE. These studies largely demonstrate fibrinolysis to prevent hemodynamic decompensation and improve RV function compared to standard anticoagulation. However, this comes at the cost of an increased bleeding risk and without a proven benefit on mortality.[13, 43, 47, 50] One specific study, PEITHO, the largest randomized, placebo-controlled trial of fibrinolysis for intermediate-risk PE to date, randomized 1005 patients to either tenecteplase or placebo, in addition to standard unfractionated heparin. Treatment with tenectaplase was found to be associated with reduced hemodynamic decompensation at 7 days $(1.6 \%$ vs. $5.0 \%, \mathrm{p}=0.002)$ with increased rates of major extracranial bleeding $(6.3 \%$ vs. $1.2 \%, \mathrm{p}<0.001)$ and stroke $(2.4 \%$ vs. $0.2 \%, \mathrm{p}=0.004)$. There was no difference between groups in all-cause mortality at 7 or 30 days, nor was there a difference in long-term outcomes including residual dyspnea and RV dysfunction.[14] When taken together, the evidence suggests that the use of thrombolytic therapy in intermediate-risk PE is likely best approached on a case-by-case basis rather than a one-size-fits-all approach, taking into consideration each patient's specific clinical scenario and individual risk.

\section{Thrombolytic therapy in low-risk PE}

Low-risk PE is characterized by normotension and no signs of RV dysfunction. This group carries the best overall prognosis including the lowest short-term mortality rate, with a simplified pulmonary embolism severity index (sPESI) score of 0 being associated with an estimated 30-day mortality risk of $1.1 \%$.[51] Thrombolytic therapy is not recommended for use in low-risk PE. [4-6]

\section{Long-term outcomes:}

\section{Mortality}

Long-term follow-up from the PEITHO trial (median of 37.8 months) demonstrated no difference in long-term survival with fibrinolysis vs. standard anticoagulation alone in intermediate-risk PE (long-term mortality rate of $20.3 \%$ vs. $18 \%, p=0.43$ ). It is important to note that long-term follow-up was not performed by all PEITHO sites and therefore was only available in 709 patients, or approximately two-thirds of the entire randomized population ( $\mathrm{n}=1006)$.[52] Several systematic review and meta-analyses have further investigated the impact of thrombolytic therapy on mortality with varying results. [37-48] It is important to note that while these studies benefit from relatively large numbers of pooled data, there exists much heterogeneity between studies, including the severity of PE, the type of thrombolytic used, and the specific dosing and administration patterns. One such SRMA, conducted by Chaterjee et al., included results from a total of 16 trials of 2115 patients (including 8 trials of 1775 patients with intermediate-risk PE). This analysis reported thrombolytic therapy to be associated with lower all-cause mortality both overall (OR 0.53 , 
95\% CI 0.32-0.88) as well as in the intermediate-risk subgroup alone (OR 0.48, 95\% CI 0.25-0.92). This mortality benefit was not significant, however, in the subgroup of patients $>65$ years of age. [38] It is important to note that mortality outcomes were reported at varying time intervals, with a mean duration of follow-up of 81.7 days. According to authors, a further sensitivity analysis including outcomes from individual trials with the longest available mortality follow-up demonstrated consistent findings. A similar SRMA investigating the impact of fibrinolysis on mortality included 15 trials of 2057 patients. This study reported overall reduced mortality in those treated with fibrinolysis (OR $0.59,95 \%$ CI 0.36-0.96). However, when studies of high-risk PE were excluded, this mortality benefit was no longer significant (OR 0.64, 95\% CI 0.35-1.17).[43] A separate SRMA of only intermediate-risk PE (including 7 studies of 1631 patients) reported a non-significant trend toward decreased all-cause 30-day mortality.[47]

\section{RV function}

$\mathrm{RV}$ dysfunction is a poor prognostic factor in acute $\mathrm{PE}$, with an RV:LV ratio $>1.0$ increasing the probability of PE-related death five-fold.[53, 54] If patients survive the acute PE event, many are plagued by residual RV dysfunction leading to persistent dyspnea, exercise intolerance and decreased quality of life measures, in what has been previously termed the "post-PE syndrome." [55, 56] Multiple studies have demonstrated treatment with thrombolytic therapy to be of short-term benefit to RV function, more rapidly relieving RV strain and improving pulmonary arterial pressures. One particular study evaluated the effect of tenecteplase vs. placebo on RV dysfunction in 51 patients with intermediate-risk PE, measuring RV:LV end-diastolic dimension ratio on echocardiography at 24 hours.[13] This study reported treatment with tenecteplase to be significantly associated with reduction of RV dysfunction. A similar study of 101 hemodynamically stable patients with acute PE randomized to alteplase or heparin alone, finding those receiving alteplase to have significantly improved RV wall motion, decreased RV end-diastolic area and improved pulmonary perfusion at 24 hours. [50] However, it remains less clear whether this initial benefit to RV function is maintained in the long-term. A systematic review and metaanalysis conducted by Sista et al. investigated persistent RV dysfunction, functional capacity limitation, exercise intolerance, and quality of life impairment following PE. Results from 26 studies (including 3671 patients) suggested treatment with thrombolysis to be associated with lower, but not statistically significant, risk of RV dysfunction and moderate functional impairment at >3-month mean follow-up. [57] However, long-term outcomes from the PEITHO trial, again with a median follow-up period of 37.8 months, demonstrated no differences in persistent dyspnea or functional limitation, residual pulmonary hypertension or RV dysfunction, or rates of chronic thromboembolic pulmonary hypertension (CTEPH) between those receiving tenecteplase and placebo.[52]

\section{Venous thromboembolism recurrence}

Incidence of recurrent venous thromboembolism (VTE) remains high, with one recent study reporting a 53\% cumulative incidence of recurrence after the maximum follow-up period of 10 years.[58] VTE recurrence increases one's risk of mortality, prolongs the duration of anticoagulation therapy and worsens patient quality of life.[55, 56, 59] Results from several 
meta-analyses suggest that treatment with thrombolytic therapy reduces risk of recurrent VTE, but whether this contributes to higher quality of life remains uncertain.[38, 43, 47]

\section{Special considerations:}

\section{Cardiopulmonary arrest}

The role of thrombolytic therapy in undifferentiated cardiopulmonary arrest has been previously studied, as well as in arrest secondary to confirmed or presumed PE. One relatively large study of adults with witnessed out-of-hospital cardiac arrest of unknown etiology randomized patients to either tenecteplase or placebo during cardiopulmonary resuscitation (CPR). The primary endpoint of this study was 30-day survival, with several secondary endpoints including hospital admission, return of spontaneous circulation (ROSC), 24-hour survival, survival to hospital discharge and neurologic outcome. This study terminated prematurely for futility after enrolling 1050 patients (525 to each arm), finding no significant differences in endpoints between groups and increased ICH in those given tenecteplase.[60] Similarly, another study of patients with pulseless electrical activity (PEA) of unknown or presumed cardiovascular cause randomized 233 patients to either $100 \mathrm{mg}$ tPA or placebo, again reporting no evidence of benefit in the primary outcome of survival to hospital discharge.[61] However, a retrospective study of patients with cardiac arrest after known PE found a significantly higher rate of ROSC in those patients treated with thrombolysis $(n=21)$ vs. those not receiving thrombolysis $(n=21), p=0.03$. Only 2 of these 21 patients treated with thrombolytic therapy survived to hospital discharge.[62] Finally, in the PEAPETT study, 23 patients with PEA and cardiopulmonary arrest due to confirmed massive PE were all given $50 \mathrm{mg}$ of tPA during CPR. ROSC was achieved in all but 1 of these patients, with 20 of the 23 still alive at 22-month follow-up.[63] Taken together, the evidence suggests that thrombolysis may be attempted in cases of cardiopulmonary arrest due to known or presumed PE, while there is likely no benefit in arrest of unclear etiology. If attempted, most studies support the administration of an IV bolus dose of 50 to $100 \mathrm{mg}$ of tPA, although specific dosing guidelines remain varied. Once thrombolysis is administered, it is recommended to continue CPR for a period of time long enough to allow for the thrombolytic medication to become efficacious, with guideline recommendations ranging from 15 to 90 minutes, before terminating resuscitation attempts.[6, 60-64] An additional worthwhile consideration involves the potential use of mechanical circulatory support, such as extracorporeal membrane oxygenation (ECMO), in these highest risk patients, as preceding administration of systemic thrombolysis increases the risk of bleeding complications resulting from cannulation. If at a facility capable of rapidly initiating ECMO, and if allowed by the clinical situation at-hand, it would be most ideal to refrain from administering systemic thrombolysis prior to cannulation. Although no clear guidelines exist on the timing of when such a procedure may be safely attempted following the administration of systemic thrombolysis, safe and successful ECMO has been previously described in this scenario.[65-68]

\section{Catheter-directed thrombolysis}

Catheter-directed thrombolysis (CDT) has been suggested as a potentially safer and more efficacious route of administration for thrombolysis, as it may be able to deliver a lower 
dose, more targeted therapy. Although there is a lack of randomized data directly comparing CDT to systemic fibrinolysis, observational data suggests CDT may confer a lower bleeding risk.[69] A recent study conducted by Beyer et al. utilized a large, administrative claims database to compare outcomes in patients undergoing CDT vs. systemic thrombolysis, finding significantly lower rates of major bleeding and ICH in those treated with CDT.[70] The OPTALYSE PE trial attempted to determine the lowest optimal tPA dose and delivery using ultrasound-facilitated catheter-directed thrombolysis (USCDT) for intermediate-risk PE, utilizing four different regimens ranging from $4 \mathrm{mg}$ to $12 \mathrm{mg}$ of tPA per lung and $2 \mathrm{hr}$ to $6 \mathrm{hr}$ infusion durations. This study reported that treatment with USCDT using lower-dose tPA and a shorter delivery duration was associated with improved RV function and reduced clot burden with an overall low rate of major bleeding, although there was $1 \mathrm{ICH}$ event.[71] Several other trials have compared the use of CDT vs. standard anticoagulation with heparin alone in the treatment of both high-risk and intermediate-risk PE, generally reporting significant improvement in hemodynamic parameters (i.e. RV:LV ratio) without increased risk of bleeding complications or additional safety outcomes.[72-75] Despite these advancements, the 2016 CHEST and 2019 European Society of Cardiology guidelines provide no specific recommendation of CDT in routine use. The guidelines tend to take a pragmatic approach, recognizing that providers and patients are likely to choose CDT therapy in patients with acute high-intermediate risk or high-risk PE who have one of the following: (1) a high bleeding risk, (2) failed systemic thrombolysis, or (3) shock that is likely to cause death before systemic thrombolysis can take effect.[5, 6] These guideline recommendations reflect the need for continued investigation to better understand the impact of CDT on additional outcomes of interest left largely unaddressed by current evidence, particularly mortality.

\section{Reduced-dose thrombolysis}

To date, there has been no prospective trial to determine the optimal dose of systemic tPA for the treatment of acute PE. It has been suggested that reduced-dose thrombolysis may reduce bleeding events while still being sufficiently efficacious. For example, the use of half-dose tPA (50 mg over 2 hours) could be particularly appealing in patients considered to be at higher risk of bleeding. These patients may include the elderly, diabetic patients, those of lower body weight and pregnant women, as well as patients with other relative contraindications to systemic fibrinolysis. A SRMA conducted by Zhang et al. sought to better assess the efficacy and safety of low-dose tPA in the treatment of acute PE. This paper included three studies comparing low-dose $(0.6 \mathrm{mg} / \mathrm{kg}$, maximum $50 \mathrm{mg}$ infusion over 2 hours) with standard dose (100 mg infusion over 2 hours) tPA, finding higher rates of major bleeding events in those treated with standard dose tPA without differences in all-cause mortality or efficacy endpoints.[48] However, contrary to these findings, a repeated metaanalysis of the same data failed to find benefit of reduced-dose tPA.[46] Further, a large administrative database study compared the use of $50 \mathrm{mg}$ tPA ( $\mathrm{n}=699$ ) vs. $100 \mathrm{mg}$ tPA $(n=3069)$ in acute $P E$, utilizing propensity matching analysis as those in the half-dose group were less likely to require vasopressor therapy or invasive ventilation at baseline. This study reported half-dose alteplase to be associated with an increased need for treatment escalation (requiring additional systemic thrombolysis or catheter treatment), with similar mortality and bleeding rates.[76] The 2013 MOPETT trial randomized 121 patients with moderate PE 
to "safe dose" tPA $(0.5 \mathrm{mg} / \mathrm{kg}$, maximum $50 \mathrm{mg}$, given as $10 \mathrm{mg}$ bolus and remainder as infusion over 2 hours) plus anticoagulation or anticoagulation alone. This study reported the use of "safe dose" tPA to be associated with a significant reduction in the incidence of pulmonary hypertension and the composite end point of pulmonary hypertension and recurrent $\mathrm{PE}$ at 28 months compared to anticoagulation alone. There was no significant difference in all-cause mortality, and no bleeding occurred in either group.[77] Current evidence remains limited and suggests the need for further investigation to determine the optimal tPA dosing regimen to balance the benefits and risks of thrombolytic therapy.

\section{Pregnancy}

Acute pulmonary embolism remains one of the leading causes of maternal death in highincome countries. Pregnancy is associated with a hypercoagulable state and therefore confers a higher risk of VTE, increasing throughout pregnancy. In general, low-molecular weight heparin (LMWH) is the treatment of choice in pregnancy as it does not cross the placenta and is well-tolerated overall.[6] However, the most appropriate treatment strategy in pregnant patients with PE of higher severity is less clear. Pregnancy, and the associated early post-partum period, is considered to be a relative contraindication to fibrinolysis.[4-6] Risks of fibrinolysis include bleeding complications, including maternal or fetal hemorrhage, teratogenesis and fetal loss.

The overall quality of evidence regarding the use of fibrinolysis in pregnancy remains low, as there are understandably no randomized controlled trials in this group.[78] A recent systematic review of severe PE occurring during pregnancy or within 6 weeks after delivery and treated with thrombolysis or thrombectomy reported outcomes for 127 cases. Of these cases, at least $83 \%$ were characterized as "massive" and $23 \%$ had an associated cardiac arrest event. The overall survival rate following thrombolysis was $94 \%$, compared to $86 \%$ following surgical thrombectomy. Fibrinolysis was associated with a $17.5 \%$ incidence of major bleeding in pregnant patients and $58.3 \%$ incidence in postpartum patients. The pooled rate of fetal death was $12 \%$.[79] A separate review of 215 cases involving the use of thrombolysis in pregnancy compared systemic $(n=183)$ to catheter-directed $(n=19)$ thrombolysis, reporting lower rates of both maternal ( $4.4 \%$ vs. $14.75 \%)$ and fetal (1.65\% vs. $5.2 \%$ ) complications in systemic thrombolysis vs. CDT.[80] The 2019 European Society of Cardiology guidelines suggest that "thrombolysis or surgical embolectomy should be considered for pregnant women with high-risk PE" (Class IIa/ Level C evidence).[6] Ultimately, this decision should be made on a case-by-case basis, taking into account the clinical situation at-hand and the patient's overall risk.

\section{Disorders of fibrinolysis:}

\section{Resistance to fibrinolysis and the emerging significance of metabolic syndrome}

The fibrinolytic system includes several natural inhibitors, including plasminogen activator inhibitor type 1 (PAI-1), thrombin activatable fibrinolysis inhibitor (TAFI) and $a_{2}$ antiplasmin, which function through different mechanisms to suppress ongoing fibrinolytic activity. PAI- 1 is a serine protease inhibitor secreted by endothelial and adipose cells and is the primary inhibitor of t-PA and u-PA (urokinase). $a_{2}$-antiplasmin, also a serine protease 
inhibitor, functions as a direct inhibitor of plasmin. Following activation by thrombin, TAFI is an enzyme that removes fibrin C-terminal lysines resulting in the restriction of further tPA binding and activation of plasminogen.[81, 82] Upregulation of these inhibitors, from either inherited or acquired etiology, may lead to hypofibrinolysis. This upregulation has been previously demonstrated to be associated with prolonged clot lysis time (CLT), a measure of fibrinolytic potential, and higher risk of both primary and recurrent VTE.[83-88] One specific genetic polymorphism commonly identified as playing a possible role in hypofibrinolysis is the $4 \mathrm{G} / 5 \mathrm{G}$ polymorphism at the promoter site of the PAI- 1 gene. As the $4 \mathrm{G}$ allele is more transcriptionally active than the $5 \mathrm{G}$ allele, those with the $4 \mathrm{G} / 4 \mathrm{G}$ genotype may have higher PAI-1 expression and, resultantly, a greater thrombotic risk.[89, 90]

In addition to inherited disorders, patients may also develop an acquired hypofibrinolytic state due to an upregulation of fibrinolytic inhibitors resulting from a variety of factors including pregnancy, aging, trauma, surgery, medication-induced and several disease states. [91] Perhaps one of the more interesting factors implicated in defective fibrinolysis is the role of metabolic syndrome (MetS), given its wide prevalence currently estimated at $34 \%$ nationally. [92] MetS, commonly defined as the clinical clustering of abdominal obesity, impaired glucose metabolism, dyslipidemia and hypertension, has been previously associated with a proinflammatory and prothrombotic state.[93, 94] Although the underlying pathophysiology of metabolic syndrome is complex and not clearly defined, increased circulating levels of PAI-1 have been reported in this population, which may be the result of overproduction by ectopic adipose tissue and fatty liver.[95, 96] Relevant to the field of VTE, a recent study investigated tPA-catalyzed CLT in patients with intermediate-risk PE treated with tenecteplase, finding it to be predictive of bleeding risk and clinical outcomes. Patients with PE and short CLT had an increased risk of hemorrhage, while those with prolonged CLT had less benefit with respect to quality of life measures and exercise tolerance at 3 months, suggesting reduced treatment efficacy. Multivariate analysis demonstrated PAI-1 and TAFI to be independent predictors of CLT. Interestingly, this study also found increased clot tensile strength in patients with MetS and diabetes compared to disease-negative controls.[97] A separate study examining the effect of MetS on patients with acute PE receiving treatment with CDT reported an association between MetS and higher remaining mean pulmonary arterial pressures post-lysis, suggesting a potential underlying resistance to this therapy (Figure 1).[98] Further, a large retrospective analysis of patients with VTE found the components of MetS to be the most significant predictors of VTE recurrence, with recurrence risk increasing stepwise with the presence of each additional MetS criterion (Figure 2).[99]

\section{Conditions associated with a hyperfibrinolytic state}

In addition to iatrogenic hyperfibrinolysis occurring after the administration of thrombolytic therapy, several congenital and acquired conditions may result in chronic activation of the endogenous fibrinolytic system, leading to a hyperfibrinolytic state. These disorders are the consequence of a relative imbalance between activators and inhibitors of the fibrinolytic system, resulting in a subsequent increased bleeding tendency. Although rare, congenital hyperfibrinolysis can occur secondary to a deficiency of fibrinolytic inhibitors, either PAI-1 or $a_{2}$-antiplasmin. More commonly, patients may develop an acquired hyperfibrinolytic 
state due to a downregulation of fibrinolytic inhibitors caused by a variety of systemic conditions. Excess bleeding may occur in chronic liver disease as the result of reduced synthesis of $a_{2}$-antiplasmin. Conversely, nephrotic syndrome is often characterized by increased urinary loss of $a_{2}$-antiplasmin. In acute promyelocytic leukemia, increased consumption of $a_{2}$-antiplasmin can occur secondary to enhanced plasmin activation.

Acquired hyperfibrinolysis may also be seen in coagulopathies of severe trauma, as the result of endothelial cell tPA release due to tissue injury and the inhibition of PAI-1 associated with shock. Further, in disseminated intravascular coagulation (DIC), systemic inflammation leads to dysregulation of the coagulation cascade and a resultant consumptive coagulopathy.[100, 101]

\section{Approach to treatment in the setting of abnormal fibrinolysis}

How best to approach the treatment of acute PE, specifically the decision of whether to administer thrombolytic therapy, in patients with known or suspected impaired fibrinolytic potential remains largely hypothesis generating. It has been suggested that perhaps a biomarker-based approach aimed at better characterizing one's resistance profile could be used to identify the best candidates for tPA and to determine an individualized dose. Alternatively, targeted, adjunctive modification of risk factors for hypofibrinolysis, such as the components of MetS, at time of PE diagnosis may be beneficial to overall treatment efficacy, irrespective of the ultimate decision for or against use of thrombolytics.[102]

\section{Summary:}

The use of fibrinolytic agents in acute PE hastens the resolution of RV stain, leading to earlier hemodynamic improvement. However, this comes at the risk of increased bleeding. The strongest indication for fibrinolysis is in high-risk PE, or that characterized by sustained hypotension. Fibrinolysis is generally not recommended for routine use in intermediate-risk $\mathrm{PE}$, although most guidelines advise that it may be considered in patients with signs of acute decompensation and an overall low bleeding risk. Ultimately, treatment decisions should carefully weigh the risks and benefits of the individual clinical scenario at hand, including the overall severity, the patient's bleeding risk, and the presence of factors known to promote resistance to fibrinolysis. In conclusion, currently available data suggest the use of fibrinolysis in acute PE confers the short-term benefit of more rapid thrombus resolution and reduction in RV afterload, while no long-term benefit has been consistently demonstrated.

\section{Acknowledgments:}

All authors have read the journal's policy on conflicts of interest and authorship agreement. Indiana University has received funding from Janssen and Pfizer pharmaceuticals for investigator-initiated research by JAK. LKS is supported by the NIH K12 Program grant 5K12HL133310-03.

\section{References}

1. Browse NL and James DC, STREPTOKINASE AND PULMONARY EMBOLISM. Lancet, 1964 2(7368): p. 1039-43. [PubMed: 14206016]

2. Hansen F, et al., Urokinase--an activator of plasminogen from human urine. Experiences with intravenous application on twenty-two patients. Angiology, 1961 12: p. 367-71. [PubMed: 13711324] 
3. Collen D, Molecular mechanisms of fibrinolysis and their application to fibrin-specific thrombolytic therapy. J Cell Biochem, 1987 33(2): p. 77-86. [PubMed: 3553213]

4. Jaff MR, et al., Management of massive and submassive pulmonary embolism, iliofemoral deep vein thrombosis, and chronic thromboembolic pulmonary hypertension: a scientific statement from the American Heart Association. Circulation, 2011 123(16): p. 1788-830. [PubMed: 21422387]

5. Kearon C, et al., Antithrombotic Therapy for VTE Disease: CHEST Guideline and Expert Panel Report. Chest, 2016 149(2): p. 315-352. [PubMed: 26867832]

6. Konstantinides SV, et al., 2019 ESC Guidelines for the diagnosis and management of acute pulmonary embolism developed in collaboration with the European Respiratory Society (ERS). Eur Heart J, 2019.

7. Lijnen HR and Collen D, Fibrinolytic agents: mechanisms of activity and pharmacology. Thromb Haemost, 1995 74(1): p. 387-90. [PubMed: 8578491]

8. Lijnen HR, et al., The mechanism of plasminogen activation and fibrin dissolution by single chain urokinase-type plasminogen activator in a plasma milieu in vitro. Blood, 1989 73(7): p. 1864-72. [PubMed: 2713507]

9. Marder VJ and Sherry S, Thrombolytic therapy: current status (1). N Engl J Med, 1988 318(23): p. 1512-20. [PubMed: 3285216]

10. Ouriel K, A history of thrombolytic therapy. J Endovasc Ther, 200411 Suppl 2: p. Ii128-133. [PubMed: 15760256]

11. Tanswell P, et al., Pharmacokinetics and pharmacodynamics of tenecteplase in fibrinolytic therapy of acute myocardial infarction. Clin Pharmacokinet, 2002 41(15): p. 1229-45. [PubMed: 12452736]

12. Online Lexicomp, Online Lexi-Drugs. Wolters Kluwer Clinical Drug Information, Inc: Hudson, Ohio.

13. Becattini C, et al., Bolus tenecteplase for right ventricle dysfunction in hemodynamically stable patients with pulmonary embolism. Thromb Res, 2010 125(3): p. e82-6. [PubMed: 19833379]

14. Meyer G, et al., Fibrinolysis for patients with intermediate-risk pulmonary embolism. N Engl J Med, 2014 370(15): p. 1402-11. [PubMed: 24716681]

15. Lee HS, How safe is the readministration of streptokinase? Drug Saf, 1995 13(2): p. 76-80. [PubMed: 7576266]

16. Lynch M, et al., Overt and subclinical reactions to streptokinase in acute myocardial infarction. Am J Cardiol, 1994 74(9): p. 849-52. [PubMed: 7977112]

17. Urokinase pulmonary embolism trial. Phase 1 results: a cooperative study. Jama, 1970 214(12): p. 2163-72. [PubMed: 5536580]

18. Schafer BM, et al., Plasminogen activation in healing human wounds. Am J Pathol, 1994 144(6): p. 1269-80. [PubMed: 8203466]

19. Hoylaerts M, et al., Kinetics of the activation of plasminogen by human tissue plasminogen activator. Role of fibrin. J Biol Chem, 1982 257(6): p. 2912-9. [PubMed: 7199524]

20. Gong L, et al., Structural basis of specific inhibition of tissue-type plasminogen activator by plasminogen activators inhibitor-1. Data Brief, 2016 6: p. 550-5. [PubMed: 26909366]

21. Mutch NJ, et al., TAFIa, PAI-1 and alpha-antiplasmin: complementary roles in regulating lysis of thrombi and plasma clots. J Thromb Haemost, 2007 5(4): p. 812-7. [PubMed: 17388801]

22. Daley MJ, Murthy MS, and Peterson EJ, Bleeding risk with systemic thrombolytic therapy for pulmonary embolism: scope of the problem. Ther Adv Drug Saf, 2015 6(2): p. 57-66. [PubMed: 25922654]

23. Curtis GM, et al., Risk factors associated with bleeding after alteplase administration for pulmonary embolism: a case-control study. Pharmacotherapy, 2014 34(8): p. 818-25. [PubMed: 24854996]

24. Fiumara K, et al., Predictors of major hemorrhage following fibrinolysis for acute pulmonary embolism. Am J Cardiol, 2006 97(1): p. 127-9. [PubMed: 16377297]

25. Chatterjee $\mathrm{S}$, et al., Risk factors for intracranial haemorrhage in patients with pulmonary embolism treated with thrombolytic therapy Development of the PE-CH Score. Thromb Haemost, 2017 117(2): p. 246-251. [PubMed: 27882375] 
26. Squire IB, et al., Humoral and cellular immune responses up to 7.5 years after administration of streptokinase for acute myocardial infarction. Eur Heart J, 1999 20(17): p. 1245-52. [PubMed: 10454976]

27. Engelter ST, et al., Life-threatening orolingual angioedema during thrombolysis in acute ischemic stroke. J Neurol, 2005 252(10): p. 1167-70. [PubMed: 16184341]

28. Hill MD, et al., Hemi-orolingual angioedema and ACE inhibition after alteplase treatment of stroke. Neurology, 2003 60(9): p. 1525-7. [PubMed: 12743244]

29. Poorthuis MHF, et al., Plasma fibrinogen level as a potential predictor of hemorrhagic complications after catheter-directed thrombolysis for peripheral arterial occlusions. J Vasc Surg, 2017 65(5): p. 1519-1527.e26. [PubMed: 28274749]

30. Wang R, et al., Risk factors of hemorrhagic transformation after intravenous thrombolysis with rtPA in acute cerebral infarction. Qjm, 2019 112(5): p. 323-326. [PubMed: 30566606]

31. Kline JA, et al., Treatment of submassive pulmonary embolism with tenecteplase or placebo: cardiopulmonary outcomes at 3 months: multicenter double-blind, placebo-controlled randomized trial. J Thromb Haemost, 2014 12(4): p. 459-68. [PubMed: 24484241]

32. Goldhaber SZ, Agnelli G, and Levine MN, Reduced dose bolus alteplase vs conventional alteplase infusion for pulmonary embolism thrombolysis. An international multicenter randomized trial. The Bolus Alteplase Pulmonary Embolism Group. Chest, 1994 106(3): p. 718-24. [PubMed: 8082347]

33. Kaufman C, Kinney T, and Quencer K, Practice Trends of Fibrinogen Monitoring in Thrombolysis. J Clin Med, 2018 7(5).

34. Lee K, et al., Fibrinogen Level and Bleeding Risk During Catheter-Directed Thrombolysis Using Tissue Plasminogen Activator. Vasc Endovascular Surg, 2015 49(7): p. 175-9. [PubMed: 26462979]

35. Meyer G, Vieillard-Baron A, and Planquette B, Recent advances in the management of pulmonary embolism: focus on the critically ill patients. Ann Intensive Care, 2016 6(1): p. 19. [PubMed: 26934891]

36. Stein PD, et al., Trends in case fatality rate in pulmonary embolism according to stability and treatment. Thromb Res, 2012 130(6): p. 841-6. [PubMed: 22909825]

37. Cao Y, et al., Systematic review and meta-analysis for thrombolysis treatment in patients with acute submassive pulmonary embolism. Patient Prefer Adherence, 2014 8: p. 275-82. [PubMed: 24611003]

38. Chatterjee S, et al., Thrombolysis for pulmonary embolism and risk of all-cause mortality, major bleeding, and intracranial hemorrhage: a meta-analysis. Jama, 2014 311(23): p. 2414-21.

[PubMed: 24938564]

39. Chen $\mathrm{H}$ and Ren C, Thrombolysis versus anticoagulation for the initial treatment of moderate pulmonary embolism: a meta-analysis of randomized controlled trials. Respir Care, 2014 59(12): p. 1880-7. [PubMed: 25269681]

40. Gao GY, et al., Thrombolysis for acute intermediate-risk pulmonary embolism: a meta-analysis. Thromb Res, 2015 136(5): p. 932-7. [PubMed: 26384442]

41. Hao Q, et al., Thrombolytic therapy for pulmonary embolism. Cochrane Database Syst Rev, 2018 12: p. Cd004437. [PubMed: 30560579]

42. Liu Y, et al., Recombinant tissue plasminogen activator for hemodynamically stable patients experiencing an acute pulmonary embolism: a meta-analysis. Thromb Res, 2014 134(1): p. 50-6. [PubMed: 24824292]

43. Marti C, et al., Systemic thrombolytic therapy for acute pulmonary embolism: a systematic review and meta-analysis. Eur Heart J, 2015 36(10): p. 605-14. [PubMed: 24917641]

44. Nakamura S, et al., Impact of the efficacy of thrombolytic therapy on the mortality of patients with acute submassive pulmonary embolism: a meta-analysis. J Thromb Haemost, 2014 12: p. 1086-95. [PubMed: 24829097]

45. Riera-Mestre A, et al., Thrombolysis in hemodynamically stable patients with acute pulmonary embolism: a meta-analysis. Thromb Res, 2104. 134(6): p. 1265-71.

46. Wang TF, et al., The role of thrombolytic therapy in pulmonary embolism. Blood, 2015 125: p. 2191-9. [PubMed: 25631770] 
47. Xu Q, et al., Initial thrombolysis treatment compared with anticoagulation for acute intermediaterisk pulmonary embolism: a meta-analysis. J Thorac Dis, 2015 7(5): p. 810-21. [PubMed: 26101636]

48. Zhang Z, et al., Lower dosage of recombinant tissue-type plasminogen activator (rt-PA) in the treatment of acute pulmonary embolism: a systematic review and meta-analysis. Thromb Res, 2014 133(3): p. 357-63. [PubMed: 24412030]

49. Lankeit M, et al., Highly sensitive troponin $\mathrm{T}$ assay in normotensive patients with acute pulmonary embolism. Eur Heart J, 2010 31(15): p. 1836-44. [PubMed: 20584774]

50. Goldhaber SZ, et al., Alteplase versus heparin in acute pulmonary embolism: randomised trial assessing right-ventricular function and pulmonary perfusion. Lancet, 1993 341(8844): p. 507-11. [PubMed: 8094768]

51. Jimenez D, et al., Simplification of the pulmonary embolism severity index for prognostication in patients with acute symptomatic pulmonary embolism. Arch Intern Med, 2010 170(15): p. 1383-9. [PubMed: 20696966]

52. Konstantinides SV, et al., Impact of Thrombolytic Therapy on the Long-Term Outcome of Intermediate-Risk Pulmonary Embolism. J Am Coll Cardiol, 2017 69(12): p. 1536-1544. [PubMed: 28335835]

53. Meinel FG, et al., Predictive Value of Computed Tomography in Acute Pulmonary Embolism: Systematic Review and Meta-analysis. Am J Med, 2015 128(7): p. 747-59.e2. [PubMed: 25680885]

54. Sanchez O, et al., Prognostic value of right ventricular dysfunction in patients with haemodynamically stable pulmonary embolism: a systematic review. Eur. Heart J, 2008 29(12): p. 1569-1577. [PubMed: 18495689]

55. Klok FA, et al., The post-PE syndrome: a new concept for chronic complications of pulmonary embolism. Blood Rev, 2014 28(6): p. 221-6. [PubMed: 25168205]

56. Klok FA, van Kralingen KW, and van Dijk AP, Quality of life in long-term survivors of acute pulmonary embolism. Chest, 2010 138: p. 1432-40. [PubMed: 20495104]

57. Sista AK, et al., Persistent right ventricular dysfunction, functional capacity limitation, exercise intolerance, and quality of life impairment following pulmonary embolism: Systematic review with meta-analysis. Vasc Med, 2017 22(1): p. 37-43. [PubMed: 27707980]

58. Prandoni $\mathrm{P}$, et al., The risk of recurrent venous thromboembolism after discontinuing anticoagulation in patients with acute proximal deep vein thrombosis or pulmonary embolism. A prospective cohort study in 1,626 patients. Haematologica, 2007 92(2): p. 199-205. [PubMed: 17296569]

59. Lang IM, et al., Risk factors and basic mechanisms of chronic thromboembolic pulmonary hypertension: a current understanding. Eur Respir J, 2013 41(2): p. 462-468. [PubMed: 22700839]

60. Bottiger BW, et al., Thrombolysis during resuscitation for out-of-hospital cardiac arrest. N Engl J Med, 2008 359(25): p. 2651-62. [PubMed: 19092151]

61. Abu-Laban RB, et al., Tissue plasminogen activator in cardiac arrest with pulseless electrical activity. N Engl J Med, 2002 346(20): p. 1522-8. [PubMed: 12015391]

62. Kurkciyan I, et al., Pulmonary embolism as a cause of cardiac arrest: presentation and outcome. Arch Intern Med, 2000 160(10): p. 1529-35. [PubMed: 10826469]

63. Sharifi M, et al., Pulseless electrical activity in pulmonary embolism treated with thrombolysis (from the "PEAPETT"study). Am J Emerg Med, 2016 34(10): p. 1963-1967. [PubMed: 27422214]

64. Truhlar A, et al., European Resuscitation Council Guidelines for Resuscitation 2015: Section 4. Cardiac arrest in special circumstances. Resuscitation, 2015 95: p. 148-201. [PubMed: 26477412]

65. Al-Bawardy R, et al., Extracorporeal membrane oxygenation in acute massive pulmonary embolism: a case series and review of the literature. Perfusion, 2019 34(1): p. 22-28.

66. Friedman O, Horowitz JM, and Ramzy D, Advanced Cardiopulmonary Support for Pulmonary Embolism. Tech Vasc Interv Radiol, 2017 20(3): p. 179-184. [PubMed: 29029712]

67. Maggio P, et al., Extracorporeal life support for massive pulmonary embolism. J Trauma, 2007 62(3): p. 570-6. [PubMed: 17414330] 
68. Meneveau N, et al., Outcomes after extracorporeal membrane oxygenation for the treatment of high-risk pulmonary embolism: a multicentre series of 52 cases. Eur Heart J, 2018 39(47): p. 4196-4204. [PubMed: 30137303]

69. Piazza G, et al., A Prospective, Single-Arm, Multicenter Trial of Ultrasound-Facilitated, CatheterDirected, Low-Dose Fibrinolysis for Acute Massive and Submassive Pulmonary Embolism: The SEATTLE II Study. JACC Cardiovasc Interv, 2015 8(10): p. 1382-92. [PubMed: 26315743]

70. Beyer SE, et al., Utilization and Outcomes of Thrombolytic Therapy for Acute Pulmonary Embolism: A Nationwide Cohort Study. Chest, 2020 157(3): p. 645-653. [PubMed: 31783016]

71. Tapson VF, et al., A Randomized Trial of the Optimum Duration of Acoustic Pulse Thrombolysis Procedure in Acute Intermediate-Risk Pulmonary Embolism: The OPTALYSE PE Trial. JACC Cardiovasc Interv, 2018 11(14): p. 1401-1410. [PubMed: 30025734]

72. Engelberger RP, et al., Fixed low-dose ultrasound-assisted catheter-directed thrombolysis for intermediate and high-risk pulmonary embolism. Eur Heart J, 2015 36(10): p. 597-604. [PubMed: 24334719]

73. Hennemeyer C, et al., Outcomes of Catheter-Directed Therapy Plus Anticoagulation Versus Anticoagulation Alone for Submassive and Massive Pulmonary Embolism. Am J Med, 2019 132(2): p. 240-246. [PubMed: 30367851]

74. Kuo WT, et al., Pulmonary Embolism Response to Fragmentation, Embolectomy, and Catheter Thrombolysis (PERFECT): Initial Results From a Prospective Multicenter Registry. Chest, 2015 148(3): p. 667-673. [PubMed: 25856269]

75. McCabe JM, et al., Usefulness and safety of ultrasound-assisted catheter-directed thrombolysis for submassive pulmonary emboli. Am J Cardiol, 2015 115(6): p. 821-4. [PubMed: 25633189]

76. Kiser TH, et al., Half-Dose Versus Full-Dose Alteplase for Treatment of Pulmonary Embolism. Crit Care Med, 2018 46(10): p. 1617-1625. [PubMed: 29979222]

77. Sharifi M, et al., Moderate pulmonary embolism treated with thrombolysis (from the "MOPETT" Trial). Am J Cardiol, 2013 111(2): p. 273-7. [PubMed: 23102885]

78. Stone SE and Morris TA, Pulmonary embolism during and after pregnancy. Crit Care Med, 2005 33(10 Suppl): p. S294-300. [PubMed: 16215350]

79. Martillotti G, et al., Treatment options for severe pulmonary embolism during pregnancy and the postpartum period: a systematic review. J Thromb Haemost, 2017 15(10): p. 1942-1950. [PubMed: 28805341]

80. Ho VT, et al., Thrombolysis for Venous Thromboembolism During Pregnancy: A Literature Review. Vasc Endovascular Surg, 2018 52(7): p. 527-534. [PubMed: 29804522]

81. Mosnier LO and Bouma BN, Regulation of fibrinolysis by thrombin activatable fibrinolysis inhibitor, an unstable carboxypeptidase B that unites the pathways of coagulation and fibrinolysis. Arterioscler Thromb Vasc Biol, 2006 26(11): p. 2445-53. [PubMed: 16960106]

82. Schleef RR and Loskutoff DJ, Fibrinolytic system of vascular endothelial cells. Role of plasminogen activator inhibitors. Haemostasis, 1988 18(4-6): p. 328-41. [PubMed: 3148526]

83. Bombardier C, et al., Monitoring hypercoagulability and hypofibrinolysis following acute venous Thromboembolism in children: application of the CloFAL assay in a prospective inception cohort study. Thromb Res, 2012 130(3): p. 343-9. [PubMed: 22551977]

84. Lisman T, et al., Reduced plasma fibrinolytic potential is a risk factor for venous thrombosis. Blood, 2005 105(3): p. 1102-5. [PubMed: 15466929]

85. Meltzer ME, et al., Hypofibrinolysis as a risk factor for recurrent venous thrombosis; results of the LETS follow-up study. J Thromb Haemost, 2010 8(3): p. 605-7. [PubMed: 19995410]

86. Meltzer ME, et al., Synergistic effects of hypofibrinolysis and genetic and acquired risk factors on the risk of a first venous thrombosis. PLoS Med, 2008 5(5): p. e97. [PubMed: 18462012]

87. Reddel CJ, et al., Detection of hypofibrinolysis in stable coronary artery disease using the overall haemostatic potential assay. Thromb Res, 2013 131(5): p. 457-62. [PubMed: 23582780]

88. Traby L, et al., Prediction of recurrent venous thromboembolism by clot lysis time: a prospective cohort study. PLoS One, 2012 7(12): p. e51447. [PubMed: 23240024]

89. Francis CW, Plasminogen activator inhibitor-1 levels and polymorphisms. Arch Pathol Lab Med, 2002 126(11): p. 1401-4. [PubMed: 12421149] 
90. Sartori MT, et al., The PAI-1 gene 4G/5G polymorphism and deep vein thrombosis in patients with inherited thrombophilia. Clin Appl Thromb Hemost, 2003 9(4): p. 299-307. [PubMed: 14653439]

91. Fareed J, et al., Acquired defects of fibrinolysis associated with thrombosis. Semin Thromb Hemost, 1999 25(4): p. 367-74. [PubMed: 10548070]

92. Beltran-Sanchez H, et al., Prevalence and trends of metabolic syndrome in the adult U.S. population, 1999-2010. J Am Coll Cardiol, 2013 62(8): p. 697-703. [PubMed: 23810877]

93. Grundy SM, et al., Definition of metabolic syndrome: report of the National Heart, Lung, and Blood Institute/American Heart Association conference on scientific issues related to definition. Arterioscler Thromb Vasc Biol, 2004 24(2): p. e13-8. [PubMed: 14766739]

94. Samson SL and Garber AJ, Metabolic syndrome. Endocrinol Metab Clin North Am, 2014 43(1): p. 1-23. [PubMed: 24582089]

95. Alessi MC and Juhan-Vague I, Metabolic syndrome, haemostasis and thrombosis. Thromb Haemost, 2008 99(6): p. 995-1000. [PubMed: 18521499]

96. Dunn EJ, et al., Molecular mechanisms involved in the resistance of fibrin to clot lysis by plasmin in subjects with type 2 diabetes mellitus. Diabetologia, 2006 49(5): p. 1071-80. [PubMed: 16538489]

97. Stubblefield WB, et al., Variable Resistance to Plasminogen Activator Initiated Fibrinolysis for Intermediate-Risk Pulmonary Embolism. PLoS One, 2016 11(2): p. e0148747. [PubMed: 26866684]

98. Stewart LK, Beam DM., Casciani T, Cameron SJ, Kline JA, Effect of metabolic syndrome on mean pulmonary arterial pressures in patients with acute pulmonary emblism treated with catheterdirected thrombolysis. International Journal of Cardiology, 2020 302: p. 138-142. [PubMed: 31948673]

99. Stewart LK, Kline JA, Metabolic syndrome increases risk of venous thromboembolism recurrence after deep vein thrombosis. Blood Advances, 2020 4(1):p. 127-135. [PubMed: 31917844]

100. Chapin JC and Hajjar KA, Fibrinolysis and the control of blood coagulation. Blood Rev, 2015 29(1): p. 17-24. [PubMed: 25294122]

101. Kolev K and Longstaff C, Bleeding related to disturbed fibrinolysis. Br J Haematol, 2016 175(1): p. 12-23. [PubMed: 27477022]

102. Stewart LK, Sarmiento EJ, Kline JA, Statin use is associated with reduced risk of recurrence in patients with venous thromboembolism. American Journal of Medicine, 2020. Accepted for publication. 
a)
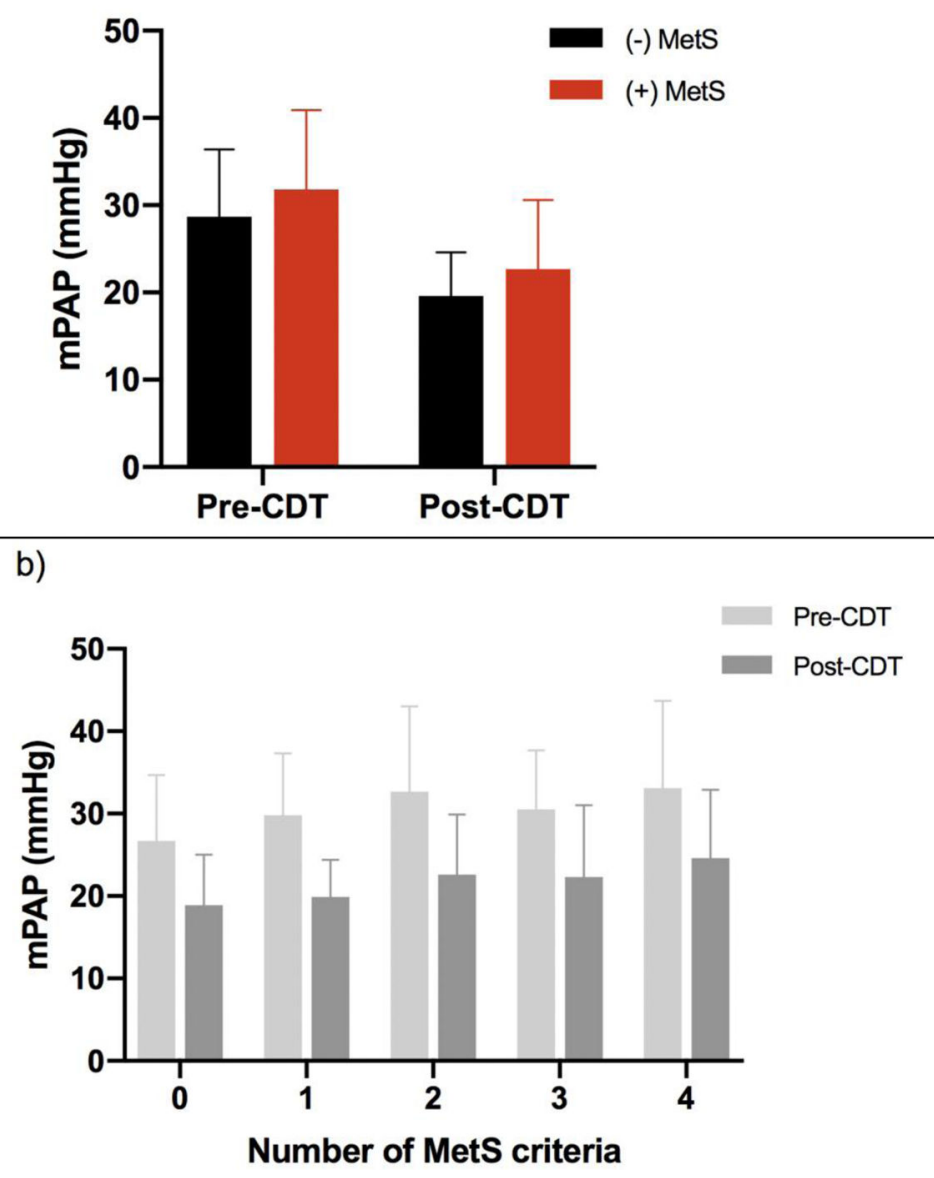

Figure 1: mPAP values in patients with acute PE measured pre- and post-CDT, stratified by (a) $+/-$ composite MetS diagnosis and (b) number of individual MetS components, displayed as means with associated standard deviations

Abbreviations: mPAP (mean pulmonary arterial pressure), PE (pulmonary embolism), CDT (catheter-directed thrombolysis), MetS (metabolic syndrome) 

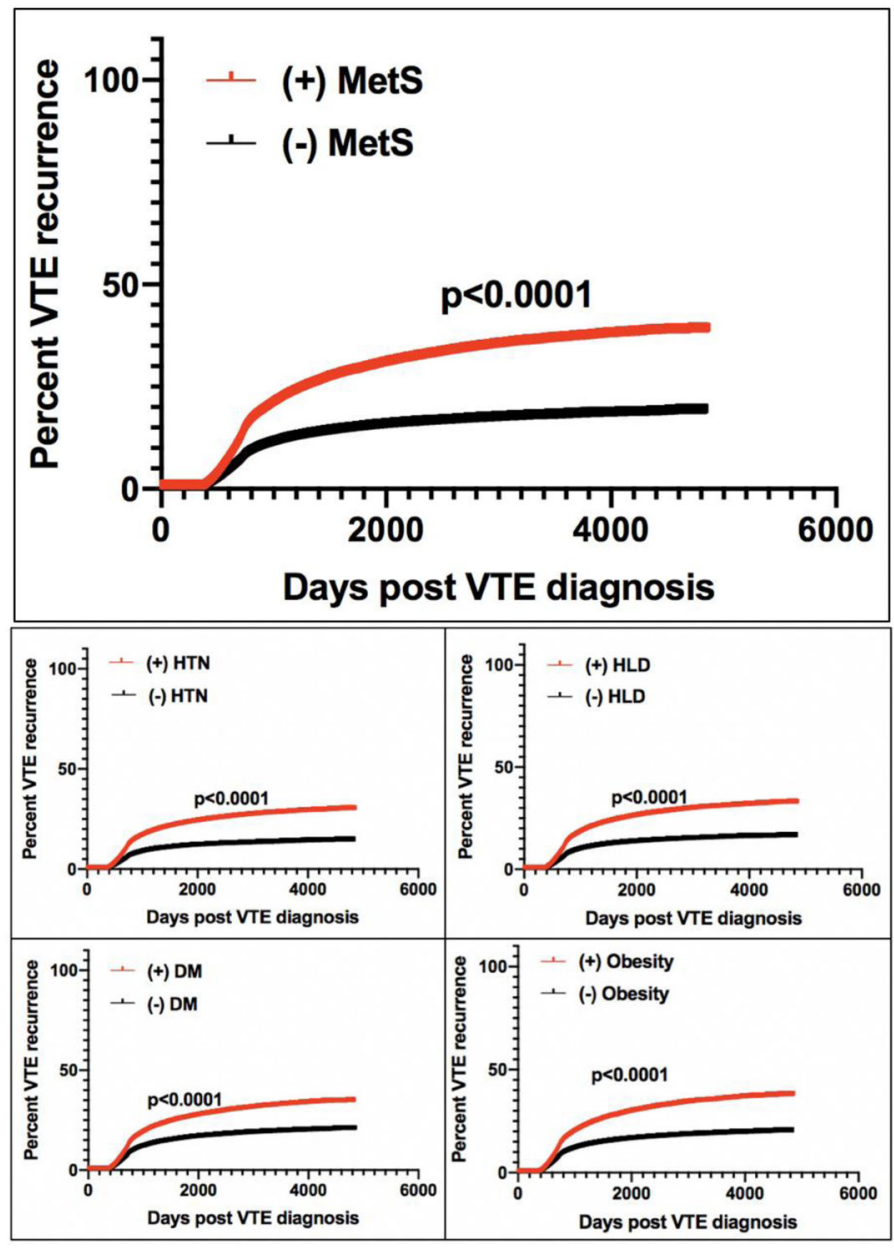

Figure 2: Kaplan-Meier curves for VTE recurrence based on +/- composite MetS diagnosis and individual MetS components

Abbreviations: VTE- venous thromboembolism, MetS- metabolic syndrome, HTNhypertension, HLD- hyperlipidemia, DM- diabetes mellitus 
Table 1:

Specific properties of commercially available fibrinolytic agents

\begin{tabular}{|c|c|c|c|c|c|}
\hline $\begin{array}{c}\text { Fibrinolytic } \\
\text { agent }\end{array}$ & $\begin{array}{l}\text { Fibrin } \\
\text { specificity }\end{array}$ & Antigenic & Dosing guidelines & $\begin{array}{l}\text { Plasma half } \\
\text { life (min) }\end{array}$ & $\begin{array}{c}\text { FDA } \\
\text { approval }\end{array}$ \\
\hline \multicolumn{6}{|l|}{$\mathbf{1}^{\text {st }}$ generation } \\
\hline Streptokinase & -- & Yes & $\begin{array}{l}250,000 \text { units IV over initial } 30 \text { minutes, followed by } 100,000 \text { units } \\
\text { IV infusion per hour for } 24 \text { hours }\end{array}$ & $12-20$ & Yes \\
\hline Urokinase & -- & No & $\begin{array}{c}\text { 4,400 units/kg IV over the initial } 10 \text { minutes, followed by } 4,400 \\
\text { units/kg IV infusion per hour for } 12 \text { hours }\end{array}$ & $7-20$ & Yes \\
\hline \multicolumn{6}{|l|}{$2^{\text {nd }}$ generation } \\
\hline Alteplase & ++ & No & $100 \mathrm{mg}$ IV infusion over 2 hours & $4-8$ & Yes \\
\hline \multicolumn{6}{|l|}{$3^{\text {rd }}$ generation } \\
\hline Tenecteplase & +++ & No & $\begin{array}{l}\text { Single weight-adjusted IV bolus injection over } \\
5 \text { to } 10 \text { seconds }\end{array}$ & $15-24$ & No \\
\hline Reteplase & + & No & $\begin{array}{c}\text { Two IV bolus injections of } 10 \text { units each, administered } 30 \text { minutes } \\
\text { apart }\end{array}$ & $11-19$ & No \\
\hline
\end{tabular}


Table 2:

\section{Contraindications to systemic fibrinolysis}

\begin{tabular}{|c|c|}
\hline Absolute contraindications & Relative contraindications \\
\hline Active bleeding & Recent bleeding \\
\hline Ischemic stroke within 3 months & Current use of anticoagulation \\
\hline Prior intracranial hemorrhage & Systolic BP $>180 \mathrm{mmHg}$ \\
\hline Brain or spinal surgery within 1 month & Diastolic BP $>110 \mathrm{mmHg}$ \\
\hline Structural cerebrovascular disease or CNS neoplasm & Traumatic or prolonged cardiopulmonary resuscitation \\
\hline Suspected aortic dissection & Ischemic stroke more than 3 months prior \\
\hline Recent head trauma with fracture or brain injury & Recent non-CNS surgery or invasive procedure \\
\hline Abdominal surgery within 7 days & Pericarditis or pericardial fluid \\
\hline & Pregnancy \\
\hline & Low body weight $(<60 \mathrm{~kg})$ \\
\hline & Age $>75$ years \\
\hline & Diabetic retinopathy \\
\hline
\end{tabular}


Table 3:

Acute pulmonary embolism risk stratification into high-, intermediate- and low-risk categories

\begin{tabular}{|c|c|c|c|c|}
\hline & $\begin{array}{c}\text { Shock or } \\
\text { hypotension } \\
\text { (SBP }<90 \\
\text { mmHg) }\end{array}$ & $\begin{array}{c}\text { Signs of RV } \\
\text { dysfunction or } \\
\text { abnormal cardiac } \\
\text { biomarkers }\end{array}$ & sPESI $^{*}$ \\
\hline \multicolumn{2}{|c|}{ High-risk } & + & Both positive (although not needed for diagnosis in presence of shock) & $\geq 1$ \\
\hline \multirow{2}{*}{ Intermediate-risk } & Intermediate-high & -- & Both positive & $\geq 1$ \\
\cline { 2 - 6 } & Intermediate-low & -- & Either one or none positive & $\geq 1$ \\
\hline \multicolumn{2}{|c|}{ Low-risk } & -- & If assessed, both negative & 0 \\
\hline
\end{tabular}

Score one point for each: age $>80$ years, history of cancer, history of chronic obstructive cardiopulmonary disease, heart rate $>110$ beats per minute, systolic blood pressure $<100 \mathrm{mmHg}$, oxygen saturation $<90 \%$ 